\title{
Reliability analysis of electronic equipment subjected to shock and vibration - A review
}

\author{
Robin Alastair Amy ${ }^{\mathrm{a}}$, Guglielmo S. Aglietti ${ }^{\mathrm{a}, *}$ and Guy Richardson ${ }^{\mathrm{b}}$ \\ ${ }^{a}$ Astronautical Research Group, University of Southampton, School of Engineering Sciences, Southampton, UK \\ ${ }^{\mathrm{b}}$ Surrey Satellite Technology Limited, Guildford, Surrey, UK
}

Received 7 January 2008

\begin{abstract}
Increasingly modern electronic equipment is expected to provide more functionality whilst still being able to withstand shock and vibration loads. The process of reliability prediction has been hampered by the complicated response and failure characteristics of electronic equipment, with the currently available methods being a compromise between accuracy and cost. A process to quickly and confidently predict the reliability of proposed electronic equipment to dynamic loads would greatly benefit industry.

This paper will illustrate the difficulties in predicting electronic equipment reliability, showing why progress has been slow, in addition to the difficulty in making a model that works across a broad range of equipment configurations. The four classes of reliability prediction methods (Handbook, Test data, Field data and Physics of Failure) will be contrasted before addressing the individual methods.

It is pertinent to note that although the majority of failures in electronic equipment are due to thermal issues, this review focuses on shock and vibration induced failures.
\end{abstract}

\section{Terminology}

BGA Ball Grid Array.

DIP Dual In-line Processor, (sometimes known as Dual In-line Package).

FE Finite Element.

PGA Pin Grid Array.

PCB Printed Circuit Board, sometimes known as a PWB (Printed Wiring Board).

PLCC Plastic Leaded Chip Carrier.

PTH Plated Through Hole, (sometimes known as Pin Through Hole).

QFP Quad Flat Pack. also known as gull wing.

SMA Shape Memory Alloys.

SMT Surface Mount Technology.

Note: In this text the term component specifically refers to any electronic device that can be soldered to a PCB, while package refers to any integrated circuit component (generally any SMT or DIP component). Finally attached component refers to any combined PCB and component system, with the intention of emphasizing the different mass and stiffness properties exhibited by an attached component.

\footnotetext{
${ }^{*}$ Corresponding author. E-mail: gsa@soton.ac.uk.
} 


\section{Background}

Shock and vibration loads imposed on a PCB will cause stresses on the PCB substrate, component packages, component leads and solder joints. These stresses are due to a combination of the bending moments in the PCB and the inertia of the component mass. In a worst case scenario these stresses may cause one of the following failure modes, PCB delamination, solder joint fracture, lead fracture or component package fracture, if any single one of these modes occurred total failure would very probably ensue. The mode of failure experienced in-service depends on the package type, PCB properties, and frequency and amplitude of both bending moments and inertial forces. The slow progress of electronic equipment reliability analysis is due to the numerous combinations of these input factors and failure modes that must be considered. The rest of this section will try to explain the complexity in trying to simultaneously consider these different input factors.

The first complicating factor to consider is the large range of package types available in modern electronics, as each package will fail due to different reasons. Heavy components will be more susceptible to inertial loads while SMT packages will be more susceptible to board curvature, within these two broad distinctions the different component types will still have vastly different failure criteria depending on mass or dimensions. The problem is further compounded by the constant stream of new components available on the market; any proposed reliability prediction method must be able to cope with new components to be of any practical use. Predicting the PCB's response is complicated by the components stiffness and mass affecting the local vibration response of the PCB, with particularly heavy or large components significantly altering the local vibration response. The PCB mechanical properties (Young's modulus and thickness) affect reliability in a non-intuitive way; as although a stiffer PCB may reduce the overall response of a PCB, it may actually locally increase the bending moments imposed on the components ${ }^{1}$. The frequency and amplitude of the local bending moment and inertial loads imposed on the package also affects the most probable failure mode, high frequency low amplitude will tend to suggest High Cycle Fatigue (HCF) ${ }^{2}$ as the failure cause. Component location on the PCB will determine whether the component stress is predominantly due to inertial loading or local bending moments. Finally the influence of human error and manufacturing variations will add a degree of deviation to the equipment reliability. With the considerable number of these input factors and their complex interactions, it is possible to understand why a fully effective method of predicting electronic equipment reliability has not yet been created.

The most recent literature review on this subject is provided by the IEEE [26]: however this review focuses mainly on the broad classifications of reliability models, such as Handbook, Field data, test data and Stress and damage (PoF) methods, and does not go into enough detail on shock and vibration failures. Foucher et al. [17] follows a similar pattern to the IEEE review, as substantial emphasis is placed on thermal failures. The previous brevity of analysis on PoF methods, especially with reference to shock and vibration failures merits further review into these areas. A review similar to the IEEE's is in the process of being compiled by the AIAA, but the scope of this review is unknown.

\section{Evolution of reliability prediction methods}

The earliest reliability prediction method developed during the 1960's is now described in MIL-HDBK-217F [44] ${ }^{3}$. This method uses databases of electronic equipment failures to give the expected useful life of a PCB incorporating certain components, and belongs to a class of reliability prediction methods known as handbook methods. Despite becoming increasingly obsolete Mil-Hdbk-217F is still in use today. The limitations and inaccuracies of the MilHdbk-217 have been well documented [42,50], which led to the development of three classes of alternative methods: Physics-of-Failure (PoF), Field data and Test data.

\footnotetext{
${ }^{1}$ Furthermore, in terms of thermally induced failures it is actually preferable to specify a more compliant PCB as this reduces the thermal stresses imposed on the package

${ }^{2}$ Low/High Cycle Fatigue, LCF relates to failures that are dominated by plastic deformation $\left(\mathrm{N}_{f}<10^{6}\right)$, whilst HCF denotes elastic deformation failures, typically $\left(\mathrm{N}_{f}>10^{6}\right)$ to failure [56].

${ }^{3}$ Mil-Hdbk-217F is the latest and final revision of the handbook, released in 1995.
} 
PoF methods predict the reliability analytically without having to resort to using historical data. All PoF methods share two characteristics of the classical method described in Steinberg [62], which first involves finding the vibration response of a PCB for a specific vibration environment and then secondarily relating individual components failure criteria to this response. An important development in PoF methods was the development of the smeared property technique for quickly creating a mathematical model of a PCB [54], this greatly reduced the complexity and time involved in accurately calculating the PCB's vibration response (see section 8.1.3). Recent developments in PoF methods have improved failure prediction for Surface Mount Technology (SMT) components; however, with the exception of Barkers method [59], these new methods are only applicable in very specific combinations of components and PCB's. There are very few methods available for large components such as transformers or large capacitors.

Field data methods improve upon the quality and implementation of the historical data used in the handbook methods. The first field data method for predicting electronic equipment reliability was documented in a 1999 paper on the HIRAP (Honeywell In-service Reliability Assessment Program) method, which was created in-house by Honeywell inc [20]. The benefits of the field data method over handbook methods are significant, in fact many similar methods have recently surfaced (REMM and TRACS [17] as well as FIDES [16]). The field data method answers the handbook methods inability to satisfactorily incorporate board-layout and operating environment into the reliability estimate. This improvement is achieved by using detailed historical failure data from boards similar to the proposed design that have experienced similar operating environments. Field data methods suffer from the requirement for an extensive database of historical failure data, where each failure type must have been correctly identified and the cause determined. This approach is suitable for companies which produce very similar equipment, with large enough batches to have a significant number of failures to assess.

The test data method for reliability has been used since the mid 1970's, and is usually partitioned into accelerated and non-accelerated tests. The basic approach is to create a test that recreates the expected operating environment as close as possible, with the intention of running the test until a failure occurs; this allows the MTBF (Mean Time Between Failure) to be predicted. If the MTBF is very high then the test duration can be reduced by using an accelerated test, this is achieved by increasing the severity of certain aspects of this test, and using existing formula to relate the failure rate in the accelerated test to the failure rate expected in service. Testing is vital for high risk components as it provides the highest confidence data; however it would be inadvisable to use it for design optimisation due to the long iteration time.

A cursory review of the work published during the 1990's would suggest that this was a period in which the field data, test data and POF methods were all competing to replace the outdated handbook methods; however, each method has its merits and limitations, and if used appropriately provides valuable results. With this in mind, the IEEE (Institute of Electrical and Electronic Engineers) recently released a standard [26] which listed all the established reliability prediction methods to date. The intention of the IEEE was to produce a guide that would ensure the engineer is informed of all available methods, along with the suitability and limitations inherent with each method. Although the IEEE approach is still in its infancy it seems to have merit, as the AIAA (American Institute of Aeronautics and Astronautics) are producing a guide referred to as S-102, which is similar to the IEEE's, but also considers the relative quality of data from each method [27]. These guides are only intended to bring together the methods that are distributed throughout the literature on the subject.

\section{Shock related failures}

The majority of past research has principally focused on random vibration as the PCB load; however the following research specifically looks at shock related failures. The methods will not be fully discussed here as they fall under the classification of PoF methods, therefore they are discussed fully in Sections 8.1 and 8.2.

Hin et al. [24] created a test board to characterise the reliability of BGA solder joints to shock. Lau et al. [36] looked at the reliability of PLCC, PQFP and QFP components to in-plane and out-of-plane shocks. Pitarresi et al. $[53,55]$ look at the failure of the failure of PC motherboards to shock loads and provides a good review of shock related literature of electronic equipment. Steinberg [62] provides a complete chapter on the design and analysis of electronic equipment subjected to shock, looking at both how to predict the shock environment and also providing 
some practical advice on how to avoid shock failure. Suhir [64,65] looked at errors in linear calculations of the response of a PCB to a shock load applied at their supports. Handbook and field data methods may consider shock related failures, but not explicitly.

\section{Handbook methods}

Of all the handbook methods available the only two that consider vibration failure are Mil-Hdbk-217 and CNET [9], Mil-Hdbk-217 being accepted as a bench-mark by the majority of manufacturers. Like all handbook methods these are empirical approaches that aim to predict the reliability of a component from field or laboratory data. Handbook methods are relatively simple to implement, in that they do not require complex mathematical modelling, only part types, part counts, application environments and other readily available parameters, these parameters are then input into a model to calculate the MTBF. Despite its advantages Mil-Hdbk-217 is increasingly falling out of favour [12, 17,42,50,51], a non-exhaustive list of the limitations are:

- The data is becoming increasingly obsolete as it was last updated in 1995 and is not relevant for new components, there is no chance of a revised model as the Defense Standards Improvement Council decided to let the method "die a natural death" [26].

- The method does not give information on the mode of failure; therefore the PCB layout can not be improved or optimised.

- The models assume that the failure is independent of design, ignoring the components location on the PCB; however the component layout is known to have a large effect on performance [50].

- The empirical data collected contains many inaccuracies, data from first-generation components with unnaturally high failure rates, defective records of operating times, repair blunders, etc, which has resulted in low confidence in the results [51].

All these disadvantages would suggest that the handbook methods should be avoided; however the limits of this method should be realised and the method only be used it when appropriate, i.e. during early design trade-off stages [46]. Unfortunately even this use should be approached with some caution as the method has not been revised since 1995. In summary, handbook methods are inherently poor at predicting mechanical reliability and should be used with caution.

\section{Test data methods}

Test data methods are the simplest of the reliability prediction methods available. A prototype of a proposed PCB design is subjected to a laboratory simulation of the vibration environment, analysis of the failure parameters (MTTF, shock spectrum) is then used to create a reliability metric [26]. The test data method should be used with respect to its advantages and disadvantages. The main advantage of test data methods is the high accuracy and confidence in the results, thus for high risk equipment the final step of the design process should always include a qualification vibration test. The disadvantage is the long time to manufacture, setup and stress a test specimen, making the method unsuitable for guiding design improvements for equipment with high failure probability, for this type of iterative design process a quicker method should be considered. The test time can be reduced by using accelerated tests, where models are available to subsequently calculate the actual service life [70,71]. These accelerated testing methods are more suited to thermal failures than vibration failures, due to the very long time for thermal failures and relatively short time for vibration. The additional complexity and error would mean these methods would generally not be applied for vibration failures, unless there are extenuating circumstances, for example, very low stresses leading to very long time to failure.

Examples of test data methods can be seen by Hart [23], Hin et al. [24], Li [37], Lau et al. [36], Shetty et al. [57], Liguore and Followell [40], Estes et al. [15], Wang et al. [67], Jih and Jung [30] and a good overall synopsis of the method is available from the IEEE [26]. 


\section{Field data methods}

As the field data method is based on failure data from previous PCB's which have experienced a particular environment; the method is only correct for PCB's which will experience the same dynamic environment. The field data method has two main aspects, building the failure database and implementing the method on a proposed design. To build the database for the field data method there must be appropriate failure data that has been collected from similar designs; this means that failure data from similar equipment must exist. The failed equipment must also have been analysed and collected properly, it is insufficient to state that a given PCB design failed after a certain number of hours, the location, failure mode and failure cause must be determined; therefore unless all previous failure data has been collected thoroughly there will be a long period of data collection required before the field data method can be used. A possible work-around for this limitation is to implement Highly Accelerated Life Testing (HALT) for the purposes of quickly building a failure rate database, although accurately determining the exact environment is difficult but vital [27] describes the second stage of implementing the field data method, using an example this paper shows how to predict the MTTF for a proposed design, where this design is modified from existing equipment for which detailed failure data exists. Other reviews of field data methods exist by various authors $[11,17,20,26]$.

\section{Physics of failure methods}

Also referred to as Stress and Damage models, PoF models are recognised by having a two stage process to predicting reliability, the first stage involves finding the response of the PCB to the dynamic loading imposed upon it, whilst the second stage takes this calculated response to provide a reliability metric. Much of the literature to date illustrates both a response prediction method and a failure criteria process; however, as the two are best described independently this review will discuss the two stages separately.

The interface between the response prediction and failure criteria stages is the response variable created in the first stage to be used in the second. The response variable has evolved from using the input acceleration at the chassis $[15,36,37,67]$, through the actual acceleration experienced by the component to account for the different vibration responses of different PCB layouts [40], and finally to looking at the local deflection [62] or local bending moments [59] experienced by the PCB local to the component. It has been noted that the failure is a function of component location on the PCB [21,38]; therefore the models that consider the local vibration response are more likely to be accurate. The choice of whether to use the local acceleration, local deflection or bending moment depends on the case at hand. If SMT components are to be used then curvature or bending moments may be most appropriate, whilst heavy components may be more likely to fail due to local accelerations. Unfortunately no research has been provided to show which type of criteria is most appropriate in which set of conditions. When choosing a response prediction method and failure criteria it is important to choose two that provide and use agreeing response variables.

It is important to consider the validity of any PoF method used, as it would be inadvisable to use any PoF method, either analytical or FE, that has not been validated against laboratory test data. Furthermore it is important to use any model within its bounds of applicability, which unfortunately limits most current PoF models to use in very specific and limited conditions.

Good examples of general discussion of the PoF methods exist by various authors [17,19,26,49].

\subsection{Response prediction}

Response prediction is concerned with using the geometry and material properties of a design to calculate the required response variable. This stage is only expected to give the overall response of the underlying PCB, not the response of the individual components. There are three main types of response prediction method, Analytical, Detailed FE models and Simplified FE models described below. These methods focus on including the stiffening and mass effects of added components; However it is important not to overlook the importance of accurately modelling the rotational stiffness at the PCB edge, as this is strongly linked with model accuracy (this is discussed in Section 8.1.4). 


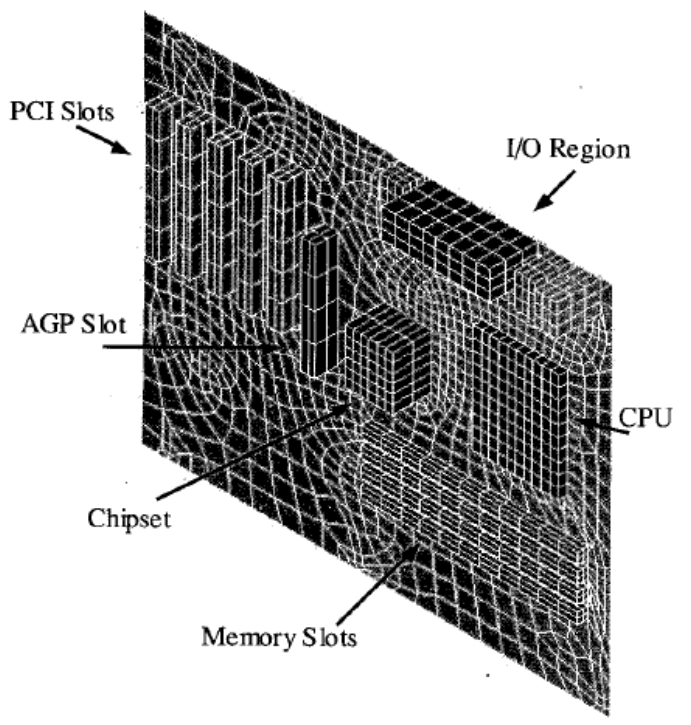

Fig. 1. Example of a detailed model of a PCB [53].

\subsubsection{Analytical response prediction}

Steinberg [62] produces the only analytical method of calculating the vibration response of a PCB. Steinberg states that the transmissibility at resonance of an electronic sub-assembly is equal to two times the square root of the resonant frequency; this statement is based on unavailable data and is unverifiable. This permits the dynamic deflection at resonance to be analytically calculated, which can be subsequently used to calculate either the dynamic load from a heavy component, or the PCB curvature. This method does not directly give the local PCB response, and is only compatible with the deflection based failure criteria provided by Steinberg. The validity of the transmissibility assumption is questionable, as Pitarresi et al. [53] measured critical damping of $2 \%$ for a computer motherboard, whilst using the given assumption would have given $3.5 \%$ (based on a natural frequency of $54 \mathrm{~Hz}$ ), which would have lead to a large underestimate of the response.

\subsubsection{Detailed FE models}

Several authors demonstrate the use of detailed FE models to calculate the PCB vibration response [30,37,53, 57,58] (Figs 1 to 3 show examples at increasing level of detail); however the use of these techniques would not be recommended for a commercial case (unless a precise prediction of the local response was absolutely vital), as the time required to build and solve such a model is excessive when simplified models produce data of appropriate accuracy much more quickly and with less effort. The time required to build and solve a detailed FE model can be reduced by using the $\mathrm{JEDEC}^{4}$ lead spring constants published by [33-35], these spring constants can be used in place of a detailed FE model of each lead ${ }^{5}$. It is also possible to implement the substructuring method (sometimes known as the superelement method) to reduce the computational time required to solve detailed models.

It should be noted that detailed FE models often blur the boundaries between response prediction and failure criteria, so the work referenced here could also fall under the failure criteria heading.

\subsubsection{Smeared FE models}

Simplified FE models reduce the time to create and solve a model by assuming that the added component mass and stiffness can be represented by simply modelling the unpopulated PCB with increased mass and stiffness (where

\footnotetext{
${ }^{4}$ Joint Electron Device Engineering Council, the semiconductor engineering standardization body of the Electronic Industries Alliance (EIA), now known as the JEDEC Solid State Technology Association.

${ }^{5}$ Although the primary aim of Kotlowitz's research is for calculating failure criteria metrics and not predicting PCB response.
} 


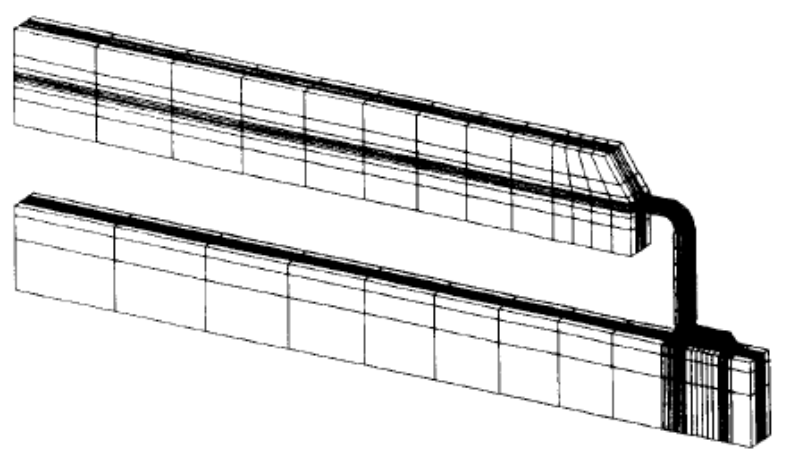

Fig. 2. Example of a detailed model of a QFP component that uses symmetry to simplify the modelling process and reduce solution time [36].

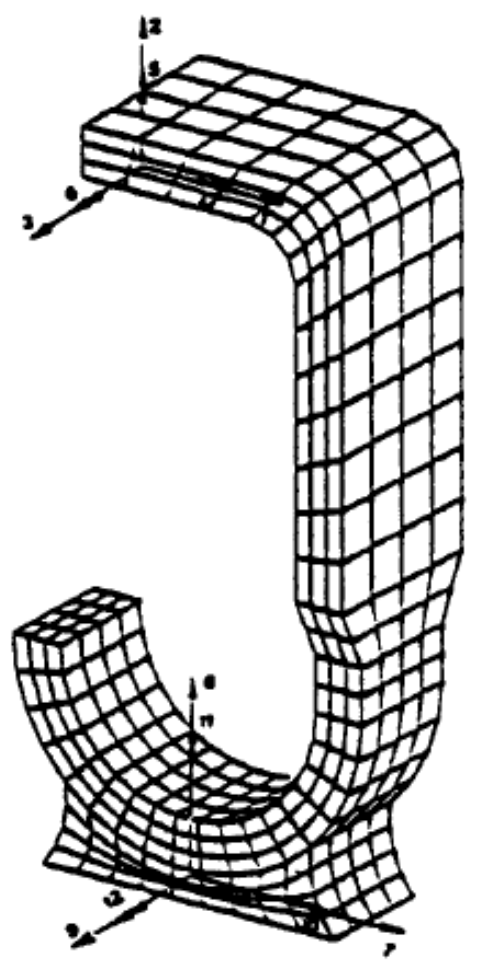

Fig. 3. Example of a detailed FE model of a J lead [6].

the mass and stiffness effects are included by locally increasing the PCB's Young's modulus). The ratio of stiffness increase can be calculated by physically cutting out an attached component and applying bend testing techniques [52].

Pitarresi et al. [52,54] consider the effect of simplifying the added mass and stiffness provided by components attached to a PCB. The first work considers a single case of a simplified FE model of a PCB, verified against experimental data. The primary area of interest of this paper is in the determination of the smeared properties, with the observation that high torsional stiffness accuracy is required for an accurate model. The second paper considers five different populated PCB's, each modelled at several different levels of property simplification. These models are compared against experimental data. The conclusion of this paper makes some enlightening observations of the correlation between mass and stiffness ratios and model accuracy. Both these papers only use the natural frequencies 
and MAC (Modal Assurance Criterion) to determine the correlation between the two models, unfortunately the error in the natural frequency can not give any information about the error in local accelerations or bending moments, also the MAC can only give a overall correlation between two mode shapes and again can not be used to give any idea of the percentage error of the acceleration or curvature.

Using a combination of numerical analysis and computer modelling, Cifuentes [10] makes the following four observations: 1, the modes modelled must contain at least $90 \%$ of the vibrating mass for accurate analysis, 2 , when board deflections are comparable to the thickness a non-linear analysis may be more appropriate than a linear one, 3 , Small errors in the location of the components can cause large errors in measuring the response and 4, the accuracy of the response measurement is more sensitive to errors in mass than stiffness.

\subsubsection{Boundary condition effects}

The PCB edge rotational stiffness greatly affects the accuracy of the calculated response [59], and depending upon the specific configuration may be of much more significance than the added component mass and stiffness. Modelling the edge rotational stiffness as zero (effectively a simply supported condition) will generally give conservative results, whilst modelling as rigidly clamped will usually underestimate results, as even the stiffest PCB clamping mechanisms is unable to provide a fully clamped edge condition.

Barker and Chen [5] validate analytical theory with experimental results to show how edge rotational stiffness affects the natural frequency of the PCB. The principal finding of this work is the strong correlation between the edge rotational stiffness and natural frequencies agreeing with theory. This also infers that large errors in modelling the edge rotational stiffness will lead to large errors in predicting the response. Although this work is principally concerned with Calmark wedgelocks, the work is applicable for modelling all types of edge constraint mechanisms.

Using experimental data Lim et al. [41] gives an example of how the edge rotational stiffness can be calculated for use in a PCB FE model; this is achieved using a method adapted from Barker and Chen [5]. This work also shows how to determine the optimum location for a single point constraint, if the maximum increase in fundamental frequencies is required.

Papers that specifically consider the effect of boundary condition modification with respect to reducing the vibration response also exist by Guo and Zhao [21]; Aglietti [2]; Aglietti and Schwingshackl [3]; Lim et al. [41].

\subsubsection{Predicting shock response}

Pitarresi et al. [53-55] use detailed FE model of PCB's to predict the response to shock and vibration, with components modelled as simple block 3D elements. These models used experimentally determined constant damping ratios to improve the prediction of response at resonance. The Shock Response Spectrum (SRS) and time-marching methods were compared for shock response prediction, with the two methods being a trade-off between accuracy and solution time.

\subsection{Failure criteria}

The failure criteria takes a measure of the PCB response and uses this to produce a failure metric, where the failure metric may be Mean Time To Failure (MTTF), cycles to failure, probability of mission success or any other of a number of failure metrics (see IEEE [26]; Jensen [28]; O'Connor [47] for a discussion of failure metrics). The many different approaches to creating this data can be conveniently split up into Analytical and Empirical categories. The empirical approaches create failure criteria data by subjecting test samples of components to a measured dynamic load. Unfortunately, due to the large range of inputs (component types, PCB thicknesses and loads) that are possible, the data published is unlikely to be directly applicable as the data is only valid in very specific cases. The analytical methods do not suffer from the same disadvantages, and have much wider applicability. 


\subsubsection{Empirical failure criteria}

As stated previously the limitation of most empirical models is that they are only valid for configurations involving similar PCB thickness, component types and input loading, which is unlikely. However the literature that is available is useful for the following reasons: they provide good examples of how to run a failure tests, they highlight the many different choices of failure metrics and they provide valuable insight into the mechanics of failure.

Li [37] created an empirical model for predicting the reliability of 272 pin BGA and 160 pin QFP packages. The failure mode studied was fatigue in the package lead near the package body, experimental results agreed well with a damage based analysis on stresses calculated using a detailed FE model(see also Li and Poglitsch [38,39]). The process gives cumulative damage for a given vibration input vibration level.

Lau et al. [36] gave the shock and vibration reliability of specific components using Weibull statistics.

Liguore and Followell [40] looked at the failure of LLCC and J leaded components, correlating local acceleration against the cycles to failure. The local acceleration is used as opposed to the chassis input acceleration; additionally the influence of temperature on the test results was also investigated. The paper also makes reference to the sensitivity of component reliability to PCB thickness.

Guo and Zhao [21] compare component reliability against local torsional curvature, in contrast to previous research which used acceleration. Using a damage based fatigue approach a FE model is favourably compared with experimental results. The paper also looks at location optimisation of components to increase reliability.

Ham and Lee [22] present a test data method for relating QFP132 lead loading to cycles to failure, based on a torsional loading input.

Estes et al. [15] look at the failure of gull wing components due to input acceleration; this is with an added dimension of thermal cycling. The components studied are CQFP 352, 208, 196, 84 and 28, as well as FP 42 and 10. As the paper is concerned with failure of components due to in-orbit vibrations, the failure is given in terms of years in Geo-stationary or Low earth orbits. The paper notes that failure of gull wing leads is more likely at locations next to the package body than at the solder joint.

Jih and Jung [30] look at failures due to inherent manufacturing defects in the solder joint. This was achieved by creating a very detailed FE model of a PCB and component to find the j-integral Power Spectral Density (PSD) for different manufacturing crack lengths.

It is suggested that the empirical methods by Ligyore and Followell [40] and Shetty and Reinikainen [58] create the most accurate and useful failure data for specific attached component configurations, which may be of use if certain variables (board thickness, component type, range of curvature) can be assumed to be constant during the design, or if the user can afford to run such specific tests.

\subsubsection{Analytical failure criteria}

SMT Corner Lead Related Models. Various researchers have explicitly considered the failure of SMT corner leads, suggesting that this is a common mode of failure. Sidharth and Barker [59] concludes an earlier series of papers, by providing a model to determine the strain of corner leads of SMT and outline leaded components. The model has less than $7 \%$ error when compared with a detailed FE model for six worst-case scenarios. The model is dependant on formula published earlier by Barker and Sidharth [4], where the deflection of an attached component subjected to a bending moment is modelled. Suhir [63] analytically looks at the stresses expected in package leads, due to locally applied bending moments. Barker and Sidharth [4] build on the work by Suhir [63] and Barker et al. [4] by considering the effect of the lead rotational stiffness. Finally Barker et al. [7] used detailed FE models to look at the effect of lead dimensional variabilities on lead fatigue life.

It is relevant to mention the work on JEDEC lead spring constants, that greatly simplifies the creation of leaded components models [33-35]. The spring constants can be used in place of a detailed model of a lead, reducing the time to build and solve a FE model or greatly simplifying the process of creating an analytical model. Using such constants in a component FE model will prevent local lead stresses from being directly calculated. Instead the overall lead strain will be given which must then be related to either local lead stresses or cycle-based lead failure criteria.

Material Fatigue Data. The majority of material failure data that exists for solders and components is principally concerned with thermal failures and there is relatively little data relevant to HCF. The principal reference on this field is provided by Sandor [56], who provides fatigue and fracture mechanics data for solders. Steinberg [62] provides 


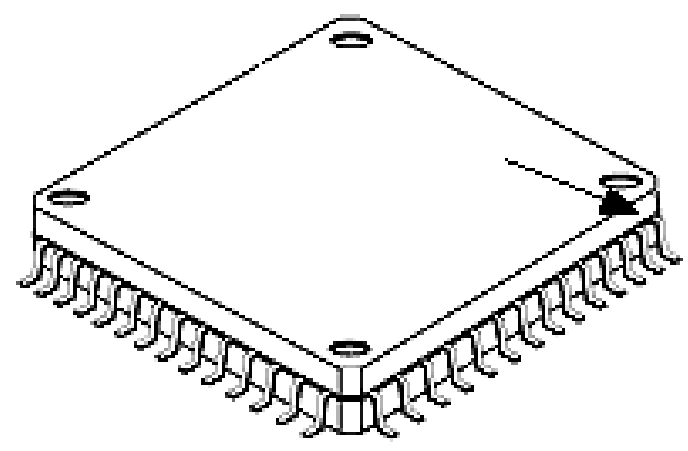

Fig. 4. Usual lead failure location for QFP components, close to package body.

fatigue data for standard solders and kovar leads. Yamada [69] looks at the fracture of solder samples. The failure of solder is complex due to the unusual properties of this material, the importance of this issue depends on the component to be tested, with QFP packages this is not as important as they usually fail at the lead, whilst with BGA, PGA and larger components the unusual properties can affect failure. Thus with QFP the fatigue properties of the lead is the most useful information. For BGA the life of solder joints subjected to instantaneous plastic deformation is more useful [14]. For larger components Steinberg [62] provides data on the pull-out stress of PTH solder joints.

Heavy Component Failure Models. The only failure models that exist for heavy components are provided by Steinberg [62], who looks at the tear out strength of PTH components, and provides an example of how to calculate the maximum allowable stress that may be placed on a PTH joint.

\subsection{Observations}

The following observations that are relevant to PoF methods have been made during the literature review:

The local response is critical in predicting component failure, as $\mathrm{Li}$ and Poglitsch [38] note that components on the edges of the PCB were less likely to fail due to local differences in bending, therefore components at different locations on the PCB will have different failure probabilities.

Curvature is considered a more important failure criteria than acceleration for SMT components, as recent papers shift attention to curvature as the primary failure criteria [38,57,62,67].

Different package types, either in number of leads or type used, are intrinsically more robust than others, regardless of the exact local environment $[15,36,38]$.

Temperature can affect the reliability of the components; Liguore and Followell [40] state that the fatigue life is highest in the temperature range from $0{ }^{\circ} \mathrm{C}$ to $65^{\circ} \mathrm{C}$, with a marked reduction below $-30^{\circ} \mathrm{C}$ and above $95^{\circ} \mathrm{C}$.

For QFP components the location where the lead joins the package (see Fig. 4) is considered as the primary failure location and not the solder joint $[15,22,38]$.

The board thickness has a definite impact on the fatigue life of a SMT components, as BGA fatigue life has shown to decrease by about $30-50$ times if the board thickness is increased from $0.85 \mathrm{~mm}$ to $1.6 \mathrm{~mm}$ (whilst maintaining constant overall curvature) [13].

The flexibility (compliance) of component leads markedly affects the reliability of peripheral leaded components [63]; however this is not a linear relationship and leads of an intermediate compliance are the least reliable.

\subsection{Software methods}

The Center for Advanced Life Cycle Engineering $\left(\mathrm{CALCE}^{\mathrm{TM}}\right)$ at the University of Maryland provides software to calculate the vibration and shock response of populated PCB's. The software (named CalcePWA TM) is a GUI 
interface that simplifies the process of running an FE model and automatically inputs the response calculation into a vibration model. The assumptions used in creating the $\mathrm{FE}$ response model are not available and the failure criteria used are taken from Steinberg [61] (Although Barkers method [48] is also assumed to be implemented). For giving general guidelines for improving equipment reliability the software is fully recommended, especially as it simultaneously considers thermally induced stresses and requires minimal specialist knowledge; however the accuracy of the failure criteria has not been validated.

\section{Equipment ruggedization}

For completeness this section will consider post-design modifications that improve electronic equipment reliability. These fall into two distint categories, those that modify the PCB boundary conditions and those that increase the damping. The primary aim of the boundary condition modifications is to reduce the PCB dynamic deflection, this can be achieved by stiffening ribs, additional supports or reducing the vibration input environment. Stiffening ribs can be useful as they raise the natural frequencies thereby reducing the dynamic deflection [62], the same applies to adding additional supports [3], although support location can also be optimised as shown by J.H. Ong and Lim [40]. Unfortunately ribs and supports would usually require a redesign of the circuit layout, therefore these methods are best considered early in the design cycle. Additionally, care should be taken to ensure that the modifications do not alter the natural frequencies to coincide with natural frequencies of the supporting structure, as this would be counter-productive. Isolation achieves higher reliability be reducing the dynamic environment transmitted to the equipment, and can either be achieved either passively or actively. Passive methods are usually simple and cheaper to implement, examples include, cable isolators [66] and recent approaches that exploit the pseudoelastic properties of Shape Memory Alloys (SMA) [32], although poorly designed isolators may actually increase response. Active methods will provide better performance over a wider range of frequencies usually at the expense of simplicity and mass, thus they are generally reserved for increasing the accuracy of very sensitive precision instruments as opposed to preventing damage. Examples of active vibration isolation include electromagnetic [60] and piezoelectric approaches $[18,43]$.

In contrast to the boundary condition modification methods, damping modification aims to reduce the peak resonant response of electronic equipment, with negligible effect on the actual natural frequencies. As with vibration isolation damping may take the passive and active approach, with similar properties of simplicity in the former approach and higher complexity and performance in the latter. Passive approaches include very simple methods such as bonding material that exhibit high damping properties to the PCB [62] through to more recent approaches such as particulate damping [68] and wideband dynamic absorbers [25]. Active vibration control is usually achieved through the use of piezoceramic elements bonded to the PCB surface [1,45].

The use of ruggedization methods depends on the specific case at hand, and should be considered carefully with relation to other methods. Applying these techniques to equipment that is not known to have reliability issues would un-necessarily increase cost and weight. However, if a design is showing failures it may be much quicker and simpler to apply a ruggedization technique than to re-design the equipment.

\section{Development opportunities}

This section details possible opportunities for improving the reliability prediction of electronic equipment, although recent advances in opto-electronics, nano-technology and packaging technology may soon limit the applicability of these suggestions.

In terms of the four main methods of reliability prediction: handbook, field data and test data can not be envisaged to allow many opportunities for improvement. The one factor that could make such methods more attractive would be completely automated low-cost manufacturing and testing methods, as this would allow a proposed design to be built and tested much more quickly than at present, with the minimum of human effort.

The PoF method does have plenty of scope for improvement. The primary area in which it could be improved is in the integration with the overall design process. Designing electronic equipment is an iterative process that brings 
together engineers with specialisation in fields of electronics, manufacturing and thermal and structural engineering. A method that automatically solves some of these issues simultaneously would reduce design iterations and save a significant amount of time, especially when the amount of inter-departmental communication is considered. The other areas of improvement for PoF methods will be divided into the response prediction and failure criteria genres.

Response prediction has two possible routes for development; either quicker detailed models or improved simplified models. With the advent of increasingly powerful computer processors the solution times of detailed FE models may become practical, whilst the build time could be reduced by software that can automatically create the desired models, eventually with the absolute minimum of human effort. Simplified FE methods could also be improved by a process to automatically create FE models, like those suggested for detailed FE methods. Automatic software is available to do this currently (CalcePWA ${ }^{\mathrm{TM}}$ ), but the technology is proprietary and the modelling assumptions unknown. Calculation of the error inherent in the different simplification techniques would be very useful; this would allow useful factors of safety to be implemented. Finally a database or method to give the increased stiffness of attached components would be useful, where these stiffness increases could be used to improve the accuracy of response models.

The creation of component failure criteria suffers from the slight variability in similar components from different manufacturers as well as the possible development of new package types, as such any method or database to give failure criteria must allow for such variability and developments. One solution would be to create a method/software to automatically build detailed FE models based on input parameters such as lead and package dimensions. Such a method could be feasible for components of generally similar shape, such as SMT or DIP components, but not for complicated irregular components such as transformers, inductors or non-standard components. The subsequent FE models could be solved for stress and combined with material failure data (S-N data, fracture mechanics or similar) to calculate the component life, although the material failure data would have to be of high quality. The FE process would need to be correlated against real test data, preferably over as wide a range of case configurations as possible.

The effort involved for such a process is relatively small when compared to the alternative of direct laboratory testing, which must run a statistically significant number of tests, at many different PCB thickness', different load intensities and directions, for the several hundred different component types available.

In terms of simple laboratory testing, there could be a method to increase the value of each test. If there were a method to calculate the relative increase in stress due to changes in some variables, for example PCB thickness or lead dimensions, then the change in component life could be subsequently estimated. Such a method could be achieved through FE analysis or analytical methods, ultimately leading to a simple formula to calculate failure criteria from existing failure data. Ultimately it is expected that a method will be created that combines all the different tools available: FE analysis, test data, analytical analysis and statistical methods, to create the most accurate failure data possible with the limited resources available.

All the separate elements of the PoF method could be improved by implementing stochastic methods into the process; allowing the effect of material and manufacturing variability to be incorporated. This would make the results more realistic in real-world cases, possibly leading to a process to create equipment that is more robust to variability, whilst minimising performance degradation (i.e. mass and cost).

Eventually such improvements may even allow real-time estimation of equipment reliability during the design process, instantaneously suggesting safer component choices, layouts or giving other advice to improve reliability, whilst simultaneously incorporating other issues: such as Electromagnetic interference (EMI), thermal and manufacturing considerations.

\section{Conclusion}

This review introduces the complexities of predicting electronic equipment reliability, follows the evolution of the four classes of analysis methods (Handbook, Field data, test data and PoF), leading to a summary and comparison of these classes. The Handbook methods are noted to be only useful for preliminary studies, Field data methods are only useful if extensive and accurate historical data is available and test data methods are vital for design qualification but poor for optimisation. PoF methods are considered in greater depth than in previous literature reviews, with the research split into response prediction and failure criteria categories. The response prediction section considers 
literature on smeared properties, boundary condition modelling, and levels of detail in FE models. The choice of response prediction method is shown to be a compromise between accuracy and time to create and solve a FE model, whilst the importance of boundary condition accuracy is again highlighted. The failure criteria section considers empirical and analytical failure criteria, SMT corner lead related models and heavy components. The empirical methods are only applicable in very specific cases although they do provide good examples of reliability testing methods, whereas analytical methods have a much wider range of applicability but are more complicated to implement. A brief discussion of current software analysis methods is included. Finally a speculative discussion on the future of reliability prediction is provided, looking at the directions in which reliability prediction methods may evolve.

\section{References}

[1] G.S. Aglietti, R.S. Langley, E. Rogers and S.B. Gabriel, An efficient model of an equipment loaded panel for active control design studies, The Journal of the Acoustical Society of America 108 (2000), 1663-1673.

[2] G.S. Aglietti, A lighter enclosure for electronics for space applications, Proceeding of Institute of Mechanical Engineers 216 (2002), $131-142$.

[3] G.S. Aglietti and C. Schwingshackl, Analysis of enclosures and anti vibration devices for electronic equipment for space applications, Proceedings of the 6th International Conference on Dynamics and Control of Spacecraft Structures in Space, Riomaggiore, Italy, (2004).

[4] D.B. Barker and Y. Chen, Modeling the vibration restraints of wedge lock card guides, ASME Journal of Electronic Packaging 115(2) (1993), 189-194.

[5] D.B. Barker, Y. Chen and A. Dasgupta, Estimating the vibration fatigue life of quad leaded surface mount components, ASME Journal of Electronic Packaging 115(2) (1993), 195-200.

[6] D.B. Barker, A. Dasgupta and M. Pecht, PWB solder joint life calculations under thermal and vibrational loading, Annual Reliability and Maintainability Symposium, 1991 Proceedings (Cat. No.91CH2966-0), 451-459.

[7] D.B. Barker, I. Sharif, A. Dasgupta and M. Pecht, Effect of SMC lead dimensional variabilities on lead compliance and solder joint fatigue life, ASME Journal of Electronic Packaging 114(2) (1992), 177-184.

[8] D.B. Barker and K. Sidharth, Local PWB and component bowing of an assembly subjected to a bending moment, American Society of Mechanical Engineers (Paper) (1993), 1-7.

[9] J. Bowles, A survey of reliability-prediction procedures for microelectronic devices, IEEE Transactions on Reliability 41(1) (1992), 2-12.

[10] A.O. Cifuentes, Estimating the dynamic behavior of printed circuit boards, IEEE Transactions on Components, Packaging, and Manufacturing Technology Part B: Advanced Packaging 17(1) (1994), 69-75.

[11] L. Condra, C. Bosco, R. Deppe, L. Gullo, J. Treacy and C. Wilkinson, Reliability assessment of aerospace electronic equipment, Quality and Reliability Engineering International 15(4) (1999), 253-260.

[12] M.J. Cushing, D.E. Mortin, T.J. Stadterman and A. Malhotra, Comparison of electronics-reliability assessment approaches, IEEE Transactions on Reliability 42(4) (1993), 542-546.

[13] R. Darveaux and A. Syed, Reliability of area array solder joints in bending, SMTA International Proceedings of the Technical Program (2000), 313-324.

[14] N.F. Enke, T.J. Kilinski, S.A. Schroeder and J.R. Lesniak, Mechanical behaviors of 60/40 tin-lead solder lap joints, Proceedings - Electronic Components Conference 12 (1989), 264-272.

[15] T. Estes, W. Wong, W. McMullen, T. Berger and Y. Saito, Reliability of class 2 heel fillets on gull wing leaded components. Aerospace Conference, Proceedings 6 (2003), 6-2517-6aC2525

[16] FIDES, FIDES Guide 2004 issue A Reliability Methodology for Electronic Systems. FIDES Group, 2004.

[17] B. Foucher, D. Das, J. Boullie and B. Meslet, A review of reliability prediction methods for electronic devices, Microelectronics Reliability 42(8) (2002), 1155-1162.

[18] J. Garcia-Bonito, M. Brennan, S. Elliott, A. David and R. Pinnington, A novel high-displacement piezoelectric actuator for active vibration control, Smart Materials and Structures 7(1) (1998), 31-42.

[19] W. Gericke, G. Gregoris, I. Jenkins, J. Jones, D. Lavielle, P. Lecuyer, J. Lenic, C. Neugnot, M. Sarno, E. Torres and E. Vergnault, A methodology to assess and select a suitable reliability prediction method for eee components in space applications, European Space Agency, (Special Publication) ESA SP (507) (2002), 73-80.

[20] L. Gullo, In-service reliability assessment and top-down approach provides alternative reliability prediction method. Annual Reliability and Maintainability, Symposium Proceedings (Cat. No.99CH36283), 1999, 365-377.

[21] Q. Guo and M. Zhao, Fatigue of SMT solder joint including torsional curvature and chip location optimization, International Journal of Advanced Manufacturing Technology 26(7-8) (2005), 887-895.

[22] S.-J. Ham and S.-B. Lee, Experimental study for reliability of electronic packaging under vibration, Experimental Mechanics 36(4) (1996), 339-344.

[23] D. Hart, Fatigue testing of a component lead in a plated through hole, IEEE Proceedings of the National Aerospace and Electronics Conference (1988), 1154-1158.

[24] T.Y. Hin, K.S. Beh and K. Seetharamu, Development of a dynamic test board for FCBGA solder joint reliability assessment in shock \& vibration. Proceedings of the 5th Electronics Packaging Technology Conference (EPTC 2003), 2003, $256-262$. 
[25] V. Ho, A. Veprik and V. Babitsky, Ruggedizing printed circuit boards using a wideband dynamic absorber, Shock and Vibration 10(3) (2003), 195-210.

[26] IEEE, IEEE guide for selecting and using reliability predictions based on ieee 1413, 2003, v+90 aC.

[27] T. Jackson, S. Harbater, J. Sketoe and T. Kinney, Development of standard formats for space systems reliability models, Annual Reliability and Maintainability Symposium, 2003 Proceedings (Cat. No.03CH37415), 269-276.

[28] F. Jensen, Electronic Component Reliability, Wiley, 1995.

[29] J.H. Ong and G. Lim, A simple technique for maximising the fundamental frequency of structures, ASME Journal of Electronic Packaging 122 (2000), 341-349.

[30] E. Jih and W. Jung, Vibrational fatigue of surface mount solder joints. IThermfl98. Sixth Intersociety Conference on Thermal and Thermomechanical Phenomena in Electronic Systems (Cat. No.98CH36208), 1998, 246-250.

[31] B. Johnson and L. Gullo, Improvements in reliability assessment and prediction methodology. Annual Reliability and Maintainability Symposium. 2000 Proceedings. International Symposium on Product Quality and Integrity (Cat. No. 00CH37055), $2000,-: 181-187$.

[32] M. Khan, D. Lagoudas, J. Mayes and B. Henderson, Pseudoelastic SMA spring elements for passive vibration isolation: part i modeling, Journal of Intelligent Material Systems and Structures 15(6) (2004), 415-441.

[33] R. Kotlowitz, Comparative compliance of representative lead designs for surface-mounted components, IEEE Transactions on Components, Hybrids, and Manufacturing Technology 12(4) (1989), 431-448.

[34] R. Kotlowitz, Compliance metrics for surface mount component lead design. 1990 Proceedings. 40th Electronic Components and Technology Conference (Cat. No. 90CH2893-6), 1990, 1054-1063.

[35] R. Kotlowitz and L. Taylor, Compliance metrics for the inclined gull-wing, spider j-bend, and spider gull-wing lead designs for surface mount components. 1991 Proceedings. 41st Electronic Components and Technology Conference (Cat. No. 91CH2989-2), 1991, 299-312.

[36] J. Lau, L. Powers-Maloney, J. Baker, D. Rice and B. Shaw, Solder joint reliability of fine pitch surface mount technology assemblies, IEEE Transactions on Components, Hybrids, and Manufacturing Technology 13(3) (1990), 534-544.

[37] R. Li, A methodology for fatigue prediction of electronic components under random vibration load, ASME Journal of Electronic Packaging 123(4) (2001), 394-400.

[38] R. Li and L. Poglitsch, Fatigue of plastic ball grid array and plastic quad flat packages under automotive vibration. SMTA International, Proceedings of the Technical Program (2001), 324-329.

[39] R. Li and L. Poglitsch, Vibration fatigue, failure mechanism and reliability of plastic ball grid array and plastic quad flat packages. Proceedings 2001 HD International Conference on High-Density Interconnect and Systems Packaging (SPIE Vol. 4428), 2001, $223-228$.

[40] S. Liguore and D. Followell, Vibration fatigue of surface mount technology (smt) solder joints. Annual Reliability and Maintainability Symposium 1995 Proceedings (Cat. No. 95CH35743), 1995, -:18-26.

[41] G. Lim, J. Ong and J. Penny, Effect of edge and internal point support of a printed circuit board under vibration, ASME Journal of Electronic Packaging 121(2) (1999), 122-126.

[42] P. Luthra, Mil-hdbk-217: What is wrong with it? IEEE Transactions on Reliability 39(5) (1990), 518.

[43] J. Marouze and L. Cheng, A feasibility study of active vibration isolation using thunder actuators, Smart Materials and Structures 11(6) (2002), 854-862.

[44] MIL-HDBK-217F. Reliability Prediction of Electronic Equipment. US Department of Defense, F edition, 1995.

[45] S.R. Moheimani, A survey of recent innovations in vibration damping and control using shunted piezoelectric transducers, IEEE Transactions on Control Systems Technology 11(4) (2003), 482-494.

[46] S. Morris and J. Reilly, Mil-hdbk-217-a favorite target. Annual Reliability and Maintainability Symposium. 1993 Proceedings (Cat. No.93CH3257-3), (1993), 503-509.

[47] P. O'Connor, Practical reliability engineering. Wiley, 1997.

[48] M. Osterman and T. Stadterman, Failure assessment software for circuit card assemblies. Annual Reliability and Maintainability. Symposium. 1999 Proceedings (Cat. No.99CH36283), 1999, 269-276.

[49] M. Pecht and A. Dasgupta, Physics-of-failure: an approach to reliable product development, IEEE 1995 International Integrated Reliability Workshop Final Report (Cat. No. 95TH8086), (1999), 1-4.

[50] M. Pecht and W.-C. Kang, A critique of mil-hdbk-217e reliability prediction methods, IEEE Transactions on Reliability 37(5) (1988), 453-457.

[51] M.G. Pecht and F.R. Nash, Predicting the reliability of electronic equipment, Proceedings of the IEEE 82(7) (1994), 992-1004.

[52] J. Pitarresi, D. Caletka, R. Caldwell and D. Smith, The smeared property technique for the FE vibration analysis of printed circuit cards, ASME Journal of Electronic Packaging 113 (1991), 250-257.

[53] J. Pitarresi, P. Geng, W. Beltman and Y. Ling, Dynamic modeling and measurement of personal computer motherboards. 52nd Electronic Components and Technology Conference 2002., (Cat. No. 02CH37345)(-), 2002, 597-603.

[54] J. Pitarresi and A. Primavera, Comparison of vibration modeling techniques for printed circuit cards, ASME Journal of Electronic Packaging 114 (1991), 378-383.

[55] J. Pitarresi, B. Roggeman, S. Chaparala and P. Geng, Mechanical shock testing and modeling of PC motherboards. 2004 Proceedings, 54th Electronic Components and Technology Conference (IEEE Cat. No. 04CH37546) 1 (2004), 1047-1054.

[56] B.I. Sandor, Solder Mechanics - A State of the Art Asssessment. The Minerals, Metals and Materials Society, 1991.

[57] S. Shetty, V. Lehtinen, A. Dasgupta, V., Halkola and T. Reinikainen, Fatigue of chip scale package interconnects due to cyclic bending, ASME Journal of Electronic Packaging 123(3) (2001), 302-308.

[58] S. Shetty and T. Reinikainen, Three- and four-point bend testing for electronic packages, ASME Journal of Electronic Packaging 125(4) (2003), 556-561.

[59] K. Sidharth and D.B. Barker, Vibration induced fatigue life estimation of corner leads of peripheral leaded components, ASME Journal of Electronic Packaging 118(4) (1996), 244-249. 
[60] J. Spanos, Z. Rahman and G. Blackwood, Soft 6- axis active vibration isolator, Proceedings of the American Control Conference 1 (1995), 412-416.

[61] D. Steinberg, Vibration Analysis for Electronic Equipment, John Wiley \& Sons, 1991

[62] D. Steinberg, Vibration Analysis for Electronic Equipment, John Wiley \& Sons, 2000.

[63] E. Suhir, Could compliant external leads reduce the strength of a surface-mounted device? 1988 Proceedings of the 38th Electronics Components Conference (88CH2600-5), 1988, 1-6.

[64] E. Suhir, Nonlinear dynamic response of a printed circuit board to shock loads applied to its support contour, ASME Journal of Electronic Packaging 114(4) (1992), 368-377.

[65] E. Suhir, Response of a flexible printed circuit board to periodic shock loads applied to its support contour, American Society of Mechanical Engineers (Paper) 59(2) (1992), 1-7.

[66] A. Veprik, Vibration protection of critical components of electronic equipment in harsh environmental conditions, Journal of Sound and Vibration 259(1) (2003), 161-175.

[67] H. Wang, M. Zhao and Q. Guo, Vibration fatigue experiments of SMT solder joint, Microelectronics Reliability 44(7) (2004), 1143-1156.

[68] Z.W. Xu, K. Chan and W. Liao, An empirical method for particle damping design, Shock and Vibration 11(5-6) (2004), 647-664.

[69] S. Yamada, A fracture mechanics approach to soldered joint cracking, IEEE Transactions on Components, Hybrids, and Manufacturing Technology 12(1) (1989), 99-104.

[70] W. Zhao and E. Elsayed, Modelling accelerated life testing based on mean residual life, International Journal of Systems Science 36(11) (1995), 689-696.

[71] W. Zhao, A. Mettas, X. Zhao, P. Vassiliou and E.A. Elsayed, Generalized step stress accelerated life model. Proceedings of 2004 International Conference on the Business of Electronic Product Reliability and Liability, 2004, 19-25. 

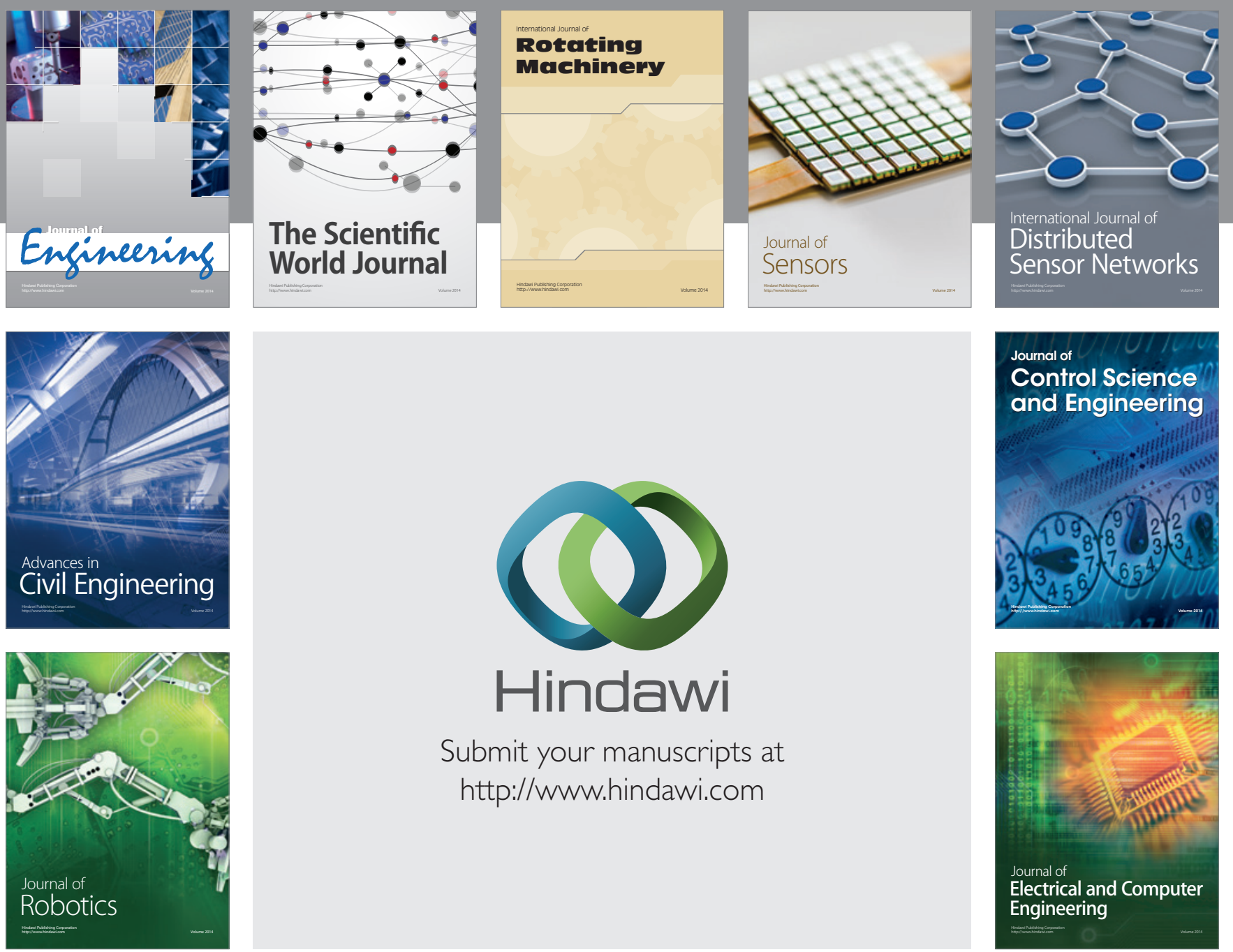

Submit your manuscripts at

http://www.hindawi.com
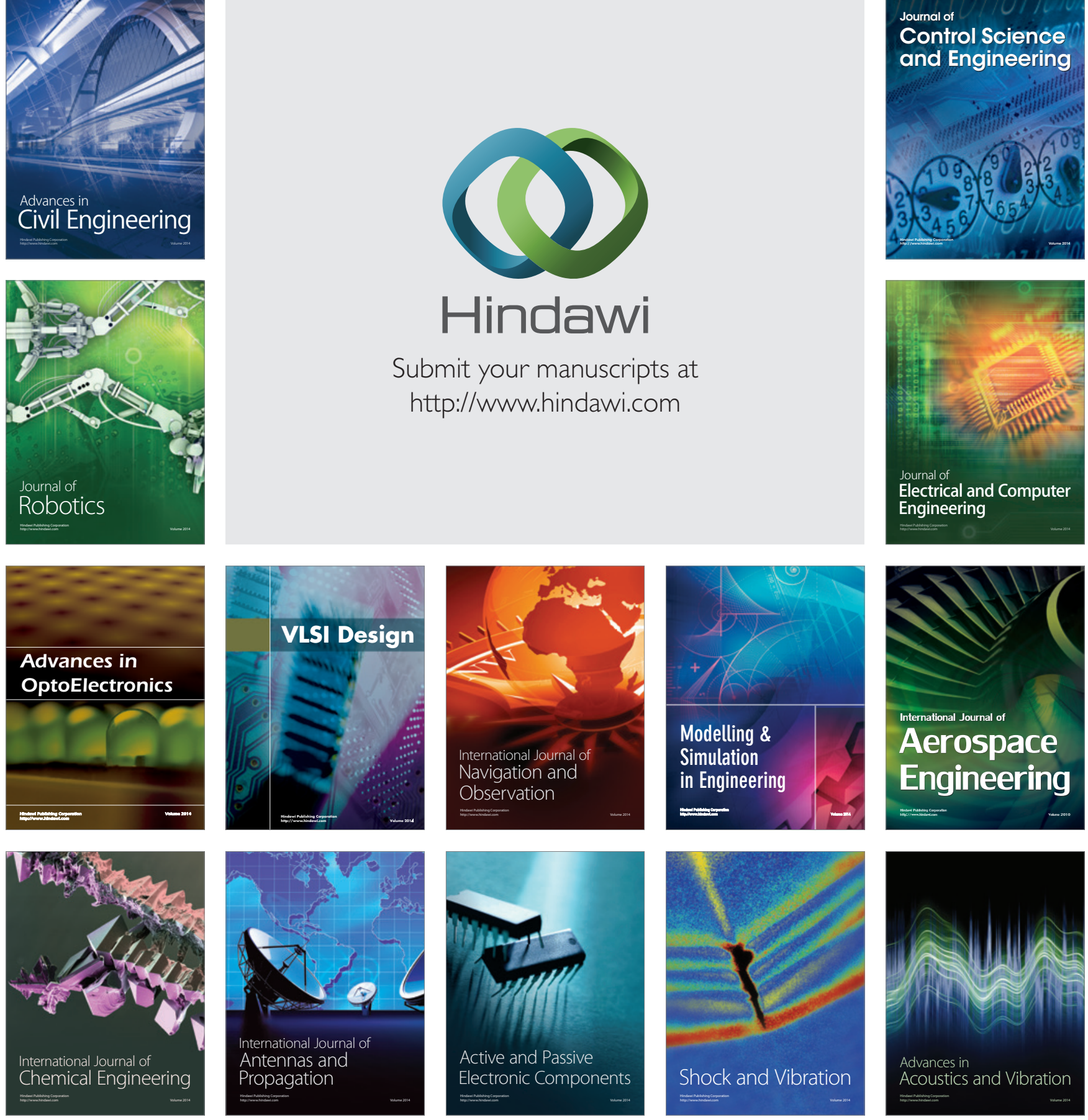